Int. J. Electrochem. Sci., 11 (2016) $6336-6346$

\title{
Flexible Supercapacitors using Liquid Phase Exfoliated Graphene with Enhanced Specific Capacitance
}

\author{
Randhir Singh ${ }^{*}$, C.C. Tripathi \\ Department of Electronics and Communication Engineering, University Institute of Engineering and \\ Technology, Kurukshetra University, Haryana, India-136119. \\ *E-mail: mr_randhir_singh@yahoo.co.in
}

doi: $10.20964 / 2016.07 .18$

Received: 5 March 2016 / Accepted: 21 April 2016 / Published: 4 June 2016

Graphene was synthesized by liquid phase exfoliation of graphite in DMF (N,N-Dimethylformamide) organic solvent. The synthesized graphene was characterized by TEM, SEM, UV-Visible and Raman spectrum. Graphene based paper electrodes were prepared by brush coating technique on A4 size paper. Four different gel electrolytes $\mathrm{PVA} / \mathrm{H}_{2} \mathrm{SO}_{4}, \mathrm{PVA} / \mathrm{KNO}_{3}, \mathrm{PVA} / \mathrm{KCL}, \mathrm{PVA} / \mathrm{KOH}$ were used for making flexible supercapacitors and their characterization was done using cyclic voltammetry $(\mathrm{CV})$ and galvanostatic charge / discharge (CD) curves. It was observed that PVA/KOH gel electrolyte exhibited maximum specific (Areal) capacitance of $30 \mu \mathrm{F} / \mathrm{cm}^{2}$ which is greater than capacitance obtained from PVA/ $\mathrm{H}_{2} \mathrm{SO}_{4}$ gel electrolyte.

Keywords: Liquid phase exfoliation; Organic Solvent; Graphene based electrodes; Flexible supercapacitor; specific capacitance.

\section{$\underline{\text { FULL TEXT }}$}

(C) 2016 The Authors. Published by ESG (www.electrochemsci.org). This article is an open access article distributed under the terms and conditions of the Creative Commons Attribution license (http://creativecommons.org/licenses/by/4.0/). 